OPEN ACCESS

Edited by:

Youmei Bao,

Yale University, United States

Reviewed by:

Longbo Zhang,

Yale University, United States

Huiguo Liu,

Huazhong University of Science and

Technology, China

Bin Peng,

Wuhan University, China

${ }^{*}$ Correspondence:

Li Zhang

zhanglith@163.com

Kuanming Huang

hkm1111@sina.com

${ }^{+}$These authors have contributed equally to this work

Specialty section: This article was submitted to

Nanobiotechnology,

a section of the journal Frontiers in Bioengineering and

Biotechnology

Received: 28 October 2021 Accepted: 13 December 2021

Published: 07 January 2022

Citation:

Tang X, Zhu J, Zhu F, Tu H, Deng A,

Lu J, Yang $M$, Dai L, Huang $K$ and

Zhang L (2022) Case Report: Primary

Pulmonary Angiosarcoma With

Brain Metastasis.

Front. Bioeng. Biotechnol. 9:803868.

doi: 10.3389/fbioe.2021.803868

\section{Case Report: Primary Pulmonary Angiosarcoma With Brain Metastasis}

\author{
Xiangjun Tang ${ }^{1 \dagger}$, Jing Zhu ${ }^{2 \dagger}$, Fangcheng $\mathrm{Zhu}^{3 \dagger}$, Hanjun $\mathrm{Tu}^{1}$, Aiping Deng ${ }^{1}$, Junti $\mathrm{Lu}^{1}$, \\ Minghuan Yang ${ }^{1}$, Longjun Dai ${ }^{1}$, Kuanming Huang ${ }^{1 *}$ and Li Zhang ${ }^{1 *}$
}

${ }^{1}$ Department of Neurosurgery, Taihe Hospital, Hubei University of Medicine, Shiyan, China, ${ }^{2}$ Department of Respiratory and Critical Care Medicine, The Central Hospital of Wuhan, Tongji Medical College, Huazhong University of Science and Technology, Wuhan, China, ${ }^{3}$ School of Basic Medical Sciences, Hubei University of Medicine, Shiyan, China

Primary pulmonary angiosarcoma (PPA) is a rare malignant vascular tumor, of which early diagnosis is challenging due to lack of specific clinical manifestations and a low level of suspicion. Here, we report a case of PPA presented with advanced brain metastasis. A 21-year-old patient with 1 week history of headache and mild cough was hospitalized for a head injury. Head MRI showed multiple intracranial lesions with brain edema. Chest CT displayed bilateral pulmonary infiltrates with mediastinal lymph node enlargement. After 2 months of anti-tuberculosis treatment, the patient was readmitted for persistent headache and cough with occasional hemosputum along with worsening pulmonary and intracranial lesions. Despite seizure prophylaxis and control of intracranial pressure and brain edema, his symptoms progressively aggravated, accompanied by cough with bloody sputum, frequent epileptic seizures, and hypotension. He eventually developed coma and died within 3 months of onset of symptoms. An autopsy confirmed PPA with brain metastasis.

Keywords: primary pulmonary angiosarcoma, brain metastasis, autopsy, diagnose, treatment

\section{INTRODUCTION}

Angiosarcoma is an uncommon malignant endothelial tumor originated from blood or lymphatic vessels, accounting for about $2 \%$ of all soft tissue sarcomas. The tumor can arise in any organ, most often in the head and neck cutaneous tissue (Young et al., 2010). It is aggressive and often multicentric, with a high rate of local recurrence and distant metastasis associated with an overall poor prognosis. The lung is usually the major metastatic site of angiosarcoma, while primary pulmonary angiosarcoma (PPA) is extremely rare (Chen et al., 2010; ObesoCarillo et al., 2013). Patients with PPA may present with various degree of respiratory symptoms, however, definite diagnosis is difficult to make based on imaging or bronchoscopy, and often requires biopsy through surgical resection. We report here an uncommon case of PPA presented with advanced brain metastasis.

\section{CASE REPORT}

The patient was a 21-year-old male non-smoker without significant past medical history other than alcohol drinking ( $150 \mathrm{~g} /$ day). One week before admission, the patient developed a swelling headache without obvious inducement. The headache progressively intensified, accompanied by minor coughs 

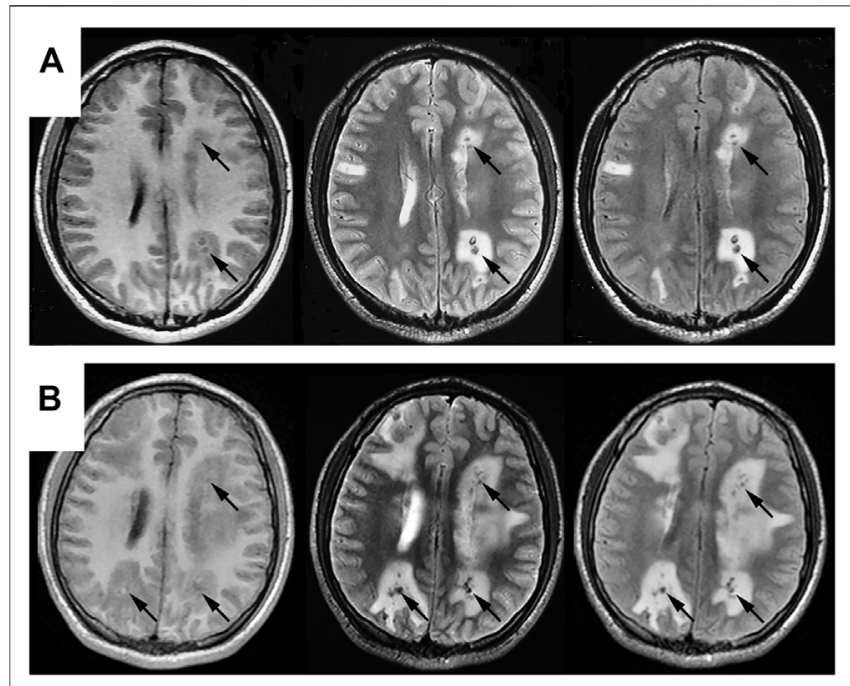

FIGURE 1 | Comparisons of cranial MRI results before (A) and after (B) admission. Both $(\mathbf{A}, \mathbf{B})$ reveal multiple nodular abnormal signals in bilateral cerebral and cerebellar hemispheres, mainly in the cortical and cortical medullary junctions, with lesions series (B) significantly larger than that in series (A). T2WI and T2flair are mainly low signals, with high signals in some lesions. T1flair shows equal and low signals, with obvious edema around the lesions.

without hemoptysis or other neurological symptoms. Two days before admission, the patient was admitted to a local hospital for head trauma and found to have multiple intracranial spaceoccupying lesions on a head CT scan. He was transferred to our hospital for further diagnosis and treatment.

On admission, the patient's vital signs were stable, mental status normal and physical examinations unremarkable. Laboratory tests showed WBC $11.42 \times 10^{9} / \mathrm{L}$, neutrophil 8.18 $\times 10^{9} / \mathrm{L}$ and eosinophil $0.51 \times 10^{9} / \mathrm{L}$, normal coagulation tests, normal tumor markers (CEA and AFP), and negative PPD test. Plain and enhanced head MRI revealed bilateral multiple intracranial lesions suggesting tuberculosis or possible metastatic tumors (Figure 1A). An enhanced CT scan of the chest showed bilateral pulmonary patchy infiltrates, most prominent in the left lower lobe dorsal segment, and an enlarged mediastinal lymph node (about $2.5 \times 1.6 \mathrm{~cm}$ ) without pleural effusion (Figure 2A). The patient was treated with intracranial pressure lowering agents and seizure prophylaxis as well as anti-tuberculosis agents and other antibiotics. One month later, patient's headache and cough remained unchanged, while repeat CT scan showed that both intracranial and pulmonary lesions had progressed significantly. The patient then underwent a bronchoscopy, which displayed bronchial inflammation and hemorrhage in the left lower lobe basal segment, as were consistent with bronchial biopsy results. No cancer cells were detected in the lavage. Considering the duration of treatment, the patient was advised to continue antituberculosis and seizures prophylaxis as an outpatient.

The patient continued suffering from intermittent headache, cough with phlegm and occasional hemosputum. When he returned for follow-up 1 month later, his head MRI showed bilateral lesions wider than those of the previous one (Figure 1B). Chest CT indicated bilateral scattered lesions, with the lesion in the left lower lobe and the mediastinal lymph node larger than before and associated with high density lesions in the lower left oblique fissure (Figure 2B). Additionally, spinal tap was performed, and examination of cerebrospinal fluid revealed colorless, transparent fluid without clots, negative for Pan's test, total protein $0.55 \mathrm{~g} / \mathrm{L}$, glucose $4.12 \mathrm{mmol} / \mathrm{L}$, and no cells or acid-fast bacilli found. While the patient continued to receive treatment for brain edema, antiinfection and seizure prophylaxis, his condition deteriorated progressively with worsening headache, frequent convulsions, hypotension, leading to coma and death. With the consent of the patient's family, an autopsy was performed, and it revealed multiple nodular lesions in the lungs and brain (Figure 3), no tumor in other organs. Immunohistochemistry of the lungs and brain displayed CK (-), Ki-67 (-), CD31 (+), CD34 (+), EMA (-) and GFAP (-). Histopathology of the lesions confirmed the diagnosis of PPA with brain metastasis (Figure 4).

\section{DISCUSSION}

PPA is a malignant tumor originating from pulmonary vascular endothelial cells and is a type of deep soft tissue angiosarcoma. The occurrence of the disease is extremely rare (Maglaras et al., 2004; Campione et al., 2009; Chen et al., 2010; ObesoCarillo et al., 2013). The average age of onset is 47.9 years-old (ranging 2-79 years) with occurrences more common in men (Drosos et al., 2019). The clinical manifestations of PPA are not specific. The most common symptoms of the disease reported so far are hemoptysis or hemosputum, cough, dyspnea, chest pain, and other non-specific symptoms such as fatigue, weight loss and fever (Ren et al., 2016). However, some patients may be asymptomatic during the course of the disease. In this case, the patient was younger than most reported patients (Drosos et al., 2019; Virarkar et al., 2019), and showed only minor coughs with occasional hemosputum, while headache due to metastasis was prominent. These factors contributed the misdiagnosis as tuberculosis.

Imaging is important in the diagnosis of pulmonary angiosarcoma, however, chest X-ray usually fails to detect fine changes of pulmonary angiosarcoma. PPA may present as multifocal or solitary nodular lesions on CT scans, with or without ground-glass changes, or with solidity changes (Ozcelik et al., 2006; Chen et al., 2010; Weissferdt and Moran, 2010). It is essential to differentiate pulmonary angiosarcoma from infectious diseases such as pneumonia and tuberculosis. Atasoy et al. reported a solitary PPA with large soft tissue masses in the left upper lobe, with mediastinal invasion and left parabronchial lymph node enlargement on the CT (Atasoy et al., 2001). At admission, our patient had pulmonary CT findings of patches in the dorsal segment of the left lower lobe, associated with ground-glass changes and enlargement of mediastinal lymph nodes, as well as multiple intracranial nodules on the cranial MRI. On the other hand, laboratory test suggested presence of infection, and bronchoscopy showed no intratracheal 
$\mathbf{A}$
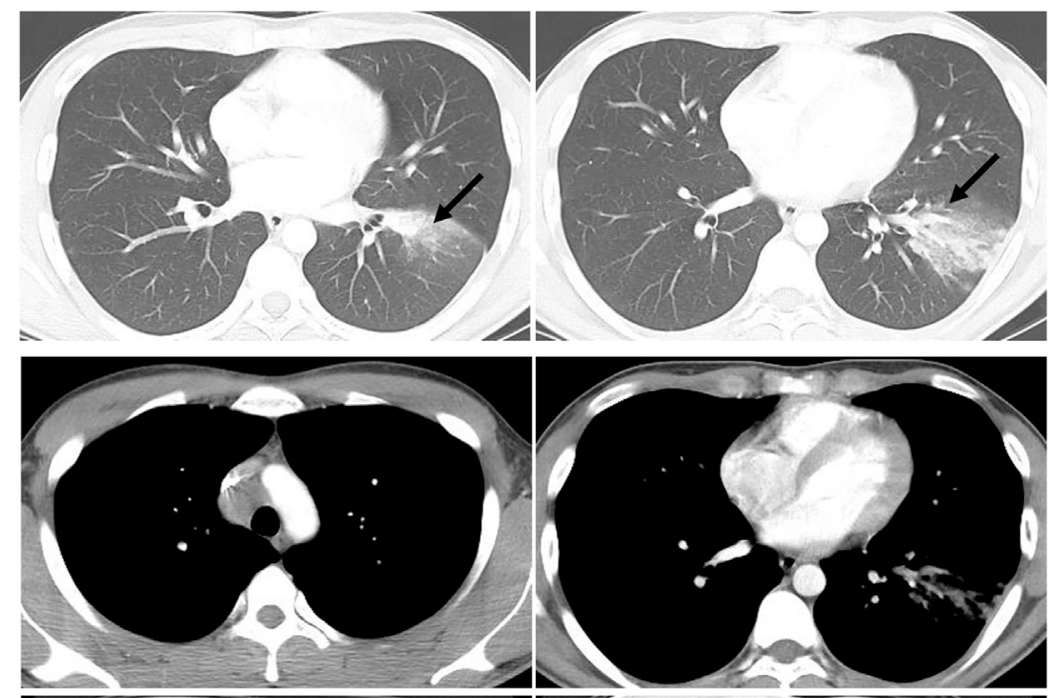

B

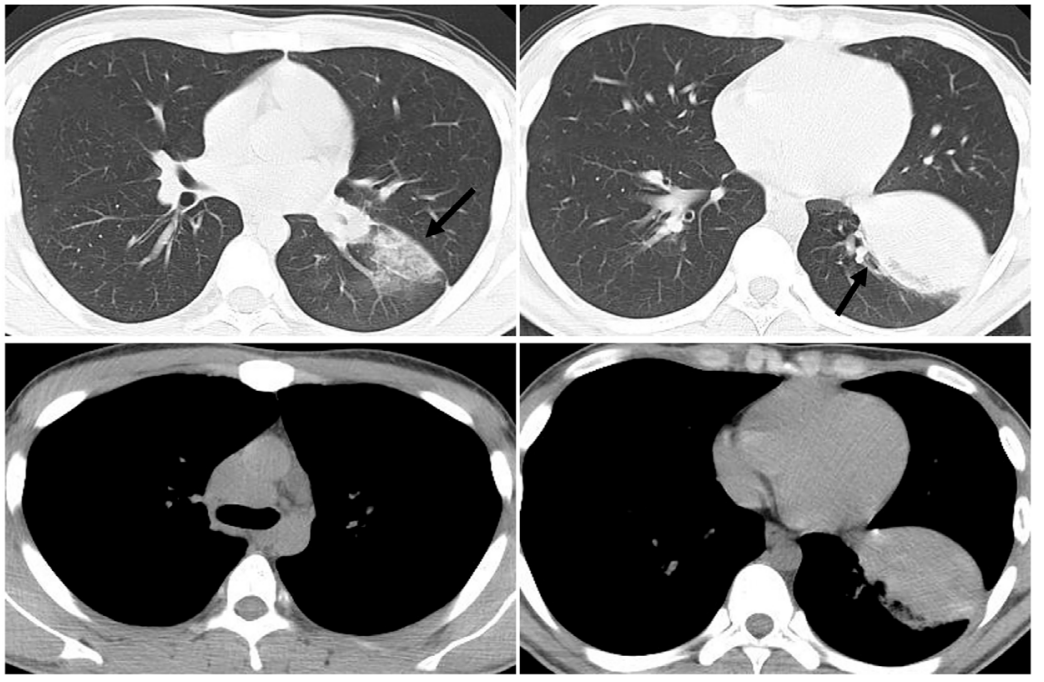

FIGURE 2 | Comparisons of chest CT results before (A) and after (B) admission. (A) shows the patchy shadow in the left lower lobe, with mediastinal lymphadenopathy. (B) shows that the left lower lung lesions are enlarged than before, with a solid shadow and lymphadenopathy.

space-occupying lesion (mass effect), pointing to infectious nature and leading to our initial misdiagnosis. However, when both pulmonary and intracranial lesions progressed rapidly under anti-tuberculosis treatment, with chest CT scan showing scattered nodules with ground-glass opacity and some solid masses with uneven density, attention should have been directed to the possibility of the highly invasive lung malignancy.

PPA accounts for $9.3 \%$ of total angiosarcomas (Drosos et al., 2019) and tends to grow latently. While local invasion and blood metastasis have often occurred at the time of detection (Maglaras et al., 2004; Grafino et al., 2016), PPA with brain metastasis was extremely rare. Our patient has this feature, with multiple intracranial metastases at the time of presentation. The main lethal factors of common brain metastatic tumors are brain edema and space-occupying effect, while our patient died of uncontrollable epilepsy and cerebral edema (Kim et al., 2015; Sun et al., 2015).
Lung biopsy and immunohistochemistry are the gold standards for the diagnosis of pulmonary angiosarcoma. Percutaneous fine needle aspiration cytology, bronchoscopic lung biopsy and thoracotomy/thoracoscopic biopsy are important means to obtain tissue specimens (Kojima et al., 2003; Campione et al., 2009; Wan Musa et al., 2010). Pathological diagnosis has also been reported through autopsy. Among them, bronchoscopy lung biopsy has a low positive rate for diagnosis of PPA, mainly because the small amount of biopsy tissue may not be sufficient to contain typical pathological features, and involvement of bronchi is not common, as were the case in our patient. Therefore, open lung biopsy is a more effective diagnostic method. Abnormal pleomorphic malignant endothelial cells are characteristic of angiosarcoma. These cells can be round, polygonal or spindle-shaped with or without epithelioid changes. In well-differentiated areas, abnormal endothelial cells form sinuses that connect to normal blood 

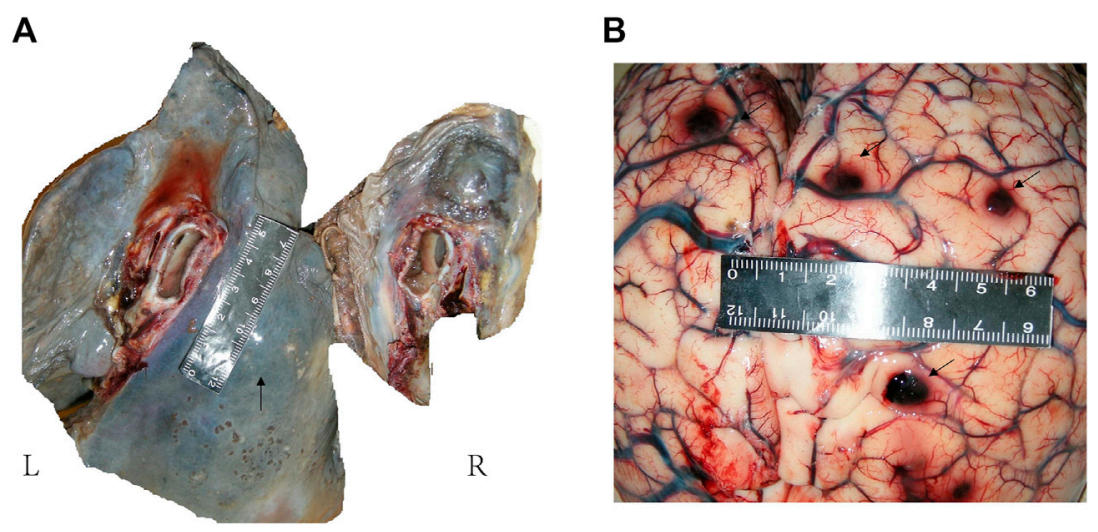

FIGURE 3 | Anatomical examination of lung and brain. (A): Gray-white hard nodules were seen in the hilar and lower lobe. There was a hard mass in the mediastinum, about $5 \mathrm{~cm} \times 2 \mathrm{~cm}$ in size. A small amount of gelatinous material was seen in the right main bronchus and pulmonary congestion was seen on section. (B): Multiple hard nodules with bleeding were seen on both sides of the cerebral hemisphere and cerebellum.
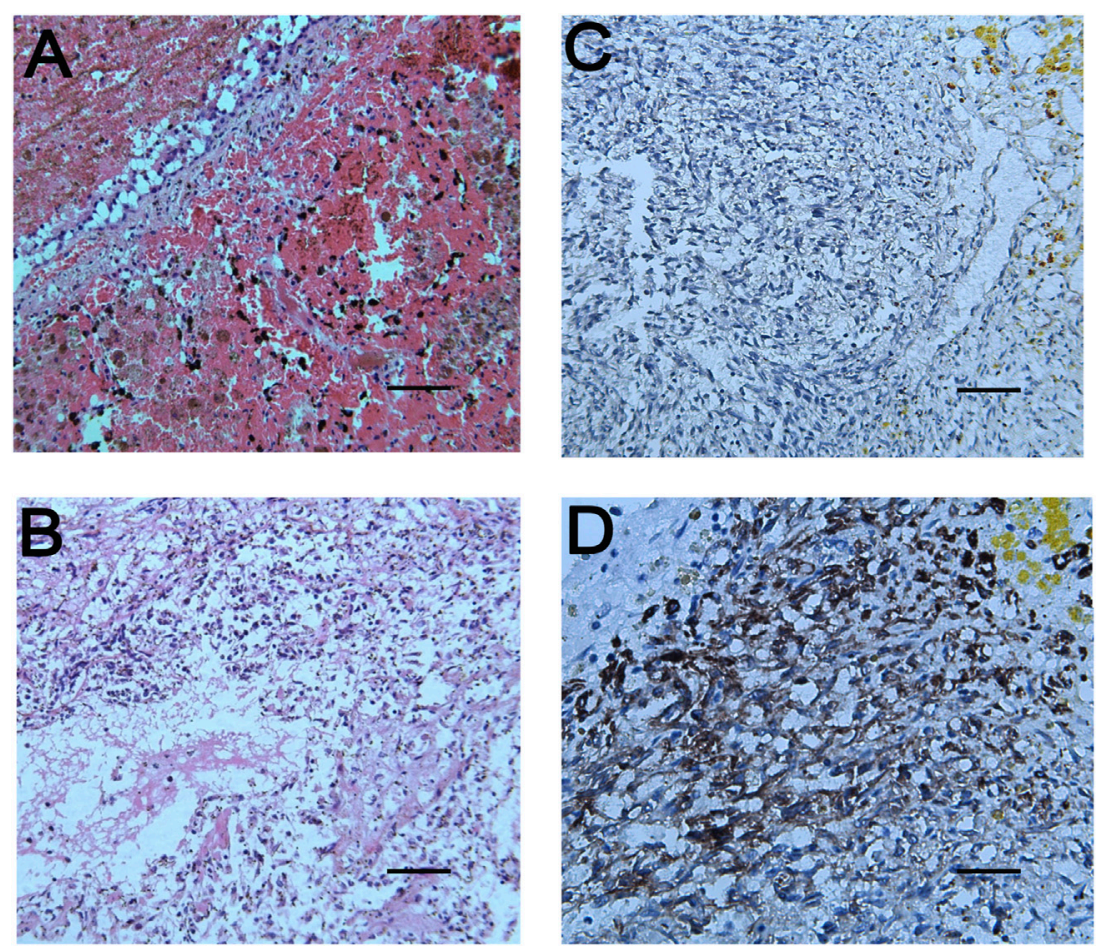

FIGURE 4 | Pathological results of lung and brain tissues. (A): The tumor cells in the pulmonary nodules are arranged in a single thin-walled vascular lumen, filled with red blood cells and accompanied by necrosis, and have a large nuclear-cytoplasmic ratio, dark-colored nuclei and atypia. (B): The lesions of the brain tissue are similar to those of the pulmonary nodules, accompanied by hemorrhage and necrosis. The surrounding brain tissue can be seen in the cuff-like infiltration of lymphocytesbased inflammatory cells around the blood vessels. Necrophages and satellite cells can also be observed. (C,D) respectively represent immunoreactivity for CD31 and CD34 on immunohistochemical examination (Magnifications: (A), ×100; (B,C), ×200; (D), ×400).

vessels. In invasive lesions, this structure becomes chaotic and does not form a well-defined cavity. In poorly differentiated regions, malignant endothelial cells form a continuous monolayer, often accompanied by epithelioid morphology. Although cytological atypia exists, the tumor cells are mainly characterized by relatively single, mitosis, necrosis and hemorrhage (Keel et al., 1999), which is consistent with the autopsy results of our patient. Immunohistochemical markers for diagnosis of pulmonary angiosarcoma include factor VIIIrelated antigen, CD34 and CD31, with factor VIII-related antigen being the most specific but not as sensitive as CD31, which is expressed in $90 \%$ of angiosarcomas (Huo et al., 2006). Our patient 
progressed rapidly and die of brain lesions scattered in multiple foci with hemorrhage, necrosis and edema. Positive CD34 and CD31 and histopathology on autopsy tissues confirmed the final diagnosis.

At present there is no unified treatment regimen for PPA. Surgical resection, radiotherapy, chemotherapy, immunotherapy and combined radiotherapy and chemotherapy have been reported in the literature (Kojima et al., 2003; Wilson et al., 2008; Chen et al., 2010; Grafino et al., 2016; Shirey et al., 2018). No treatment has been clinically proven to be effective, but treatment under certain conditions may be effective, such as early removal of focal lesions. However, the disease is often diagnosed at a late stage and beyond the indications for surgery. It has been reported that recombinant IL-2 immunotherapy combined with radiotherapy or gemcitabine combined with docetaxel may also be effective (Kojima et al., 2003). The current literature reports that the prognosis of PPA is extremely poor, with median survival time 7 months in patients with a single lesion, and 2 months in those with multiple lesions (Ren et al., 2016). The disease in this case developed rapidly and was quite aggressive, with advanced lesions beyond surgically resection at presentation, resulting in death in less than 3 months. Therefore, early detection and identification is crucial.

In conclusion, PPA is an extremely rare malignant tumor with a very poor prognosis. Because of the lack of specific clinical manifestations, timely diagnosis is difficult. The PPA patient reported here presented with early and advanced brain metastasis and was diagnosed after autopsy using histopathology and immunohistochemistry biomarkers. This case cautions us that PPA should be considered when

\section{REFERENCES}

Atasoy, Ç., Fitoz, S., Yiğit, H., Atasoy, P., Erden, I., and Akyar, S. (2001). Radiographic, CT, and MRI Findings in Primary Pulmonary Angiosarcoma. Clin. Imaging 25 (5), 337-340. doi:10.1016/s0899-7071(01)00302-3

Campione, A., Forte, G., Luzzi, L., Comino, A., Gorla, A., and Terzi, A. (2009). Pulmonary Angiosarcoma Presenting as Spontaneous Recurrent Hemothorax. Asian Cardiovasc. Thorac. Ann. 17 (1), 84-85. doi:10.1177/0218492309102544

Chen, Y.-b., Guo, L.-c., Yang, L., Feng, W., Zhang, X.-q., Ling, C.-h., et al. (2010). Angiosarcoma of the Lung: 2 Cases Report and Literature Reviewed. Lung Cancer 70 (3), 352-356. doi:10.1016/j.lungcan.2010.09.002

Drosos, E., Kalyvas, A., Komaitis, S., Skandalakis, G. P., Kalamatianos, T., Liouta, E., et al. (2019). Angiosarcoma-related Cerebral Metastases: a Systematic Review of the Literature. Neurosurg. Rev. 43, 1019-1038. doi:10.1007/ s10143-019-01127-y

Grafino, M., Alves, P., Almeida, M. M. d., Garrido, P., Hasmucrai, D., Teixeira, E., et al. (2016). Angiosarcoma of the Lung. J. Bras. Pneumol. 42 (1), 68-70. doi:10.1590/S1806-37562016000000193

Huo, L., Moran, C. A., Fuller, G. N., Gladish, G., and Suster, S. (2006). Pulmonary Artery Sarcoma. Am. J. Clin. Pathol. 125 (3), 419-424. doi:10.1309/ 9h8rhuv1jl1we0qf

Keel, S. B., Bacha, E., Mark, E. J., Nielsen, G. P., and Rosenberg, A. E. (1999). Primary Pulmonary Sarcoma: a Clinicopathologic Study of 26 Cases. Mod. Pathol. 12 (12), 1124-1131.

Kim, E. Y., Lee, H. Y., Han, J., and Choi, J. Y. (2015). Primary Pulmonary LowGrade Angiosarcoma Characterized by Mismatch between18F-FDG PET and Dynamic Contrast-Enhanced CT. Korean J. Radiol. 16 (5), 1166-1170. doi:10.3348/kjr.2015.16.5.1166 a patient's MRI shows multiple mixed signals accompanied by obvious brain edema of unknown origin. Once the disease is suspected, biopsy combined with immunohistochemistry should be used to confirm the diagnosis as soon as possible.

\section{DATA AVAILABILITY STATEMENT}

The raw data supporting the conclusions of this article will be made available by the authors, without undue reservation.

\section{ETHICS STATEMENT}

Written informed consent was obtained from the individual(s) for the publication of any potentially identifiable images or data included in this article.

\section{AUTHOR CONTRIBUTIONS}

$\mathrm{XT}$ and $\mathrm{JZ}$ wrote the manuscript; FZ, AD, JL, and MY collected the data and images; LD edited language, $\mathrm{HT}, \mathrm{KH}$, and LZ led the treatment of patients and directed the writing of the manuscript.

\section{ACKNOWLEDGMENTS}

We thank the patient and his family for helping us study this disease. We also thank Daizhong Wang, Jingjing Liu for their efforts in clinical data management.

Kojima, K., Okamoto, I., Ushijima, S., Yoshinaga, T., Kitaoka, M., Suga, M., et al. (2003). Successful Treatment of Primary Pulmonary Angiosarcoma. Chest 124 (6), 2397-2400. doi:10.1378/chest.124.6.2397

Maglaras, G. C., Katsenos, S., Kakadelis, J., Katsanos, C., Metafratzi, Z., Stefanou, D. G., et al. (2004). Primary Angiosarcoma of the Lung and Pleura. Monaldi Arch. Chest Dis. 61 (4), 234-236. doi:10.4081/monaldi.2004.687

Obeso Carillo, G. A., García Fontán, E. M., Cañizares Carretero, M. Á., and Pérez Pedrosa, A. (2013). Primary Pulmonary Angiosarcoma, an Exceptional Neoplasm with a Poor Prognosis: Reports of Two Cases and Review of the Literature. Gen. Thorac. Cardiovasc. Surg. 61 (11), 643-647. doi:10.1007/ s11748-012-0200-6

Ozcelik, C., Onat, S., Yaldiz, M., and Ozcelik, Z. (2006). Primary Epithelioid Angiosarcoma of the Lung Presenting as Pulmonary Hemorrhage. Asian Cardiovasc. Thorac. Ann. 14 (1), 69-71. doi:10.1177/021849230601400118

Ren, Y., Zhu, M., Liu, Y., Diao, X., and Zhang, Y. (2016). Primary Pulmonary Angiosarcoma: Three Case Reports and Literature Review. Thorac. Cancer 7 (5), 607-613. doi:10.1111/1759-7714.12376

Shirey, L., Coombs, D., Talwar, A., and Mickus, T. (2018). Pulmonary Epithelioid Angiosarcoma Responsive to Chemotherapy: A Case Report. Radiol. Case Rep. 13 (2), 479-484. doi:10.1016/j.radcr.2018.02.002

Sun, D., Yu, R., Chen, J., Geng, D., and Zhang, L. (2015). A Rare Case of Primary Paranasal Sinus Angiosarcoma with Pulmonary Metastasis Detected by 18FFDG PET/CT. Clin. Nucl. Med. 40 (3), 286-288. doi:10.1097/ RLU.0000000000000694

Virarkar, M., Tayyab, S., Thampy, R., Bhosale, P., and Viswanathan, C. (2019). Primary Pulmonary Angiosarcoma: Case Reports and Review of the Literature. Asian Cardiovasc. Thorac. Ann. 27 (5), 347-352. doi:10.1177/0218492319836910

Wan Musa, W. R., Abdulwakil Elraied, M. A., Phang, K. S., Kwah, Y. G., Tan, S. P., Harun, R., et al. (2010). Primary Epithelioid Angiosarcoma of the Lung 
Presenting as Left-Sided Shoulder Pain. Ann. Acad. Med. Singap 39 (8), 658-659.

Weissferdt, A., and Moran, C. A. (2010). Primary Vascular Tumors of the Lungs: a Review. Ann. Diagn. Pathol. 14 (4), 296-308. doi:10.1016/ j.anndiagpath.2010.03.001

Wilson, R., Glaros, S., Brown, R. K. J., Michael, C., and Reisman, D. (2008). Complete Radiographic Response of Primary Pulmonary Angiosarcomas Following Gemcitabine and Taxotere. Lung Cancer 61 (1), 131-136. doi:10.1016/j.lungcan.2007.12.006

Young, R. J., Brown, N. J., Reed, M. W., Hughes, D., and Woll, P. J. (2010). Angiosarcoma. Lancet Oncol. 11 (10), 983-991. doi:10.1016/S1470-2045(10) 70023-1

Conflict of Interest: The authors declare that the research was conducted in the absence of any commercial or financial relationships that could be construed as a potential conflict of interest.
The reviewer HL declared a shared affiliation, with no collaboration, with one of the authors JZ, to the handling editor at the time of the review.

Publisher's Note: All claims expressed in this article are solely those of the authors and do not necessarily represent those of their affiliated organizations, or those of the publisher, the editors and the reviewers. Any product that may be evaluated in this article, or claim that may be made by its manufacturer, is not guaranteed or endorsed by the publisher.

Copyright (c) 2022 Tang, Zhu, Zhu, Tu, Deng, Lu, Yang, Dai, Huang and Zhang. This is an open-access article distributed under the terms of the Creative Commons Attribution License (CC BY). The use, distribution or reproduction in other forums is permitted, provided the original author(s) and the copyright owner(s) are credited and that the original publication in this journal is cited, in accordance with accepted academic practice. No use, distribution or reproduction is permitted which does not comply with these terms. 